\title{
Impact on Interface Spin Polarization of Molecular Bonding to Metallic Surfaces
}

\author{
S. Javaid, ${ }^{1}$ M. Bowen, ${ }^{1}$ S. Boukari, ${ }^{1}$ L. Joly, ${ }^{1,2}$ J.-B. Beaufrand, ${ }^{1}$ Xi Chen, ${ }^{1}$ Y. J. Dappe, ${ }^{1}$ F. Scheurer, ${ }^{1}$ J.-P. Kappler, ${ }^{1}$ \\ J. Arabski, ${ }^{1}$ W. Wulfhekel, ${ }^{3}$ M. Alouani, ${ }^{1}$ and E. Beaurepaire ${ }^{1}$ \\ ${ }^{1}$ IPCMS UMR 7504 CNRS, Université de Strasbourg, 23 rue du Loess, BP 43, 67034 Strasbourg Cedex 2, France \\ ${ }^{2}$ Swiss Light Source, Paul Scherrer Institut, 5232 Villigen PSI, Switzerland \\ ${ }^{3}$ Physikalisches Institut, Karlsruhe Institute of Technology, Wolfgang-Gaede-Strasse 1, 76131 Karlsruhe, Germany
}

(Received 20 January 2010; published 13 August 2010)

\begin{abstract}
We have studied the repercussion of the molecular adsorption mechanism on the electronic properties of the interface between model nonmagnetic or magnetic metallic surfaces and metallo-organic phthalocyanines molecules (Pcs). Our intertwined x-ray absorption spectroscopy experiments and computational studies reveal that manganese $\mathrm{Pc}(\mathrm{MnPc})$ is physisorbed onto a $\mathrm{Cu}(001)$ surface and retains the electronic properties of a free molecule. On the other hand, $\mathrm{MnPc}$ is chemisorbed onto $\mathrm{Co}(001)$, leading to a dominant direct exchange interaction between the Mn molecular site and the Co substrate. By promoting an interfacial spin-polarized conduction state on the molecule, these interactions reveal an important lever to tailor the spintronic properties of hybrid organic-metallic interfaces.
\end{abstract}

DOI: 10.1103/PhysRevLett.105.077201

The injection of electron spin across and away from the interface between a ferromagnetic metal and an inorganic semiconductor is driven by wave function transmission across the spacer $[1,2]$, as well as by the electronic properties of the interface. In the latter case, the appearance of magnetic moments, induced on the atomic sites of the semiconductor due to bonding-induced exchange interactions with the ferromagnet, determine in turn which wave functions will most efficiently hybridize into evanescent states for tunneling transmission [3].

To this date, this pillar of our understanding of spin electronics has yet to be verified experimentally [4]. Meanwhile, a rapid and accelerating convergence is occurring between the fields of spin electronics and organic electronics through incremental successes at spinpolarized transport across ultrathin [5,6] or thin [7] organic layers. A number of experimental reports have also drawn attention to the peculiar electronic properties present at the interface between the metals used as spin injectors and the molecules that constitute the organic spacer layer. These reports range from ferromagnetic (FM) coupling [8] between the metal site in metallo-organic molecules [see Fig. 1(a)] and a ferromagnetic substrate, predicted to underscore an indirect exchange interaction, to the inferred presence of an electronic state within the organic layer's forbidden gap [9] that appears to drive the device's spintronic response [10]. These results are likely to reflect the impact on transport of the chemical bonds between the molecule and the ferromagnet [11].

We have studied the interface between model, metallic, $\mathrm{fcc}(001)$-oriented surfaces-either nonmagnetic $\mathrm{Cu}$ or ferromagnetic $\mathrm{Co}$, and the metallo-organic molecule manganese phthalocyanine (MnPc) [see Fig. 1(a)]. Experimental samples were grown within the preparation chamber (base pressure of $P=5 \times 10^{-10} \mathrm{mbar}$ ) of the $7 \mathrm{~T}$ cryomagnet setup maintained by the IPCMS and installed on the SIM
PACS numbers: 85.75. $-\mathrm{d}, 31.10 .+\mathrm{z}, 75.70 .-\mathrm{i}$, 78.70.Dm

beamline at the Swiss Light Source. MnPc was sublimated $\left(P=2-3 \times 10^{-9} \mathrm{mbar}\right.$ ) so as to form ultrathin films (1$5 \mathrm{ML} ; 1 \mathrm{ML}=4 \AA)$ on $\mathrm{Co}(5 \mathrm{ML}) / \mathrm{Cu}(001)$ and $\mathrm{Cu}(001)$ substrates. X-ray absorption spectroscopy (XAS) spectra were acquired in total electron yield mode at $P<2 \times$ $10^{-10}$ mbar by reversing both the circular polarity of the photons and the sign of the external magnetic field. All density functional theory calculations were carried out using the PWSCF package [12] and the generalized gradient approximation as an exchange-correlation functional with the Perdew, Burke, and Ernzerhof parametrization [13]. We used the $a b$ initio pseudopotential method with Vanderbilt ultrasoft pseudopotentials [14]. A plane-wave basis set was used with kinetic-energy cutoffs of $30 \mathrm{Ry}$ at the $\Gamma$ point of the first Brillouin zone. $\mathrm{Fcc} \operatorname{Co}(001)$ and fcc $\mathrm{Cu}(001)$ surfaces were modeled by using periodic supercells, each made of 3 atomic layers of $8 \times 8$ atoms and vacuum. The supercell lattice parameter perpendicular to

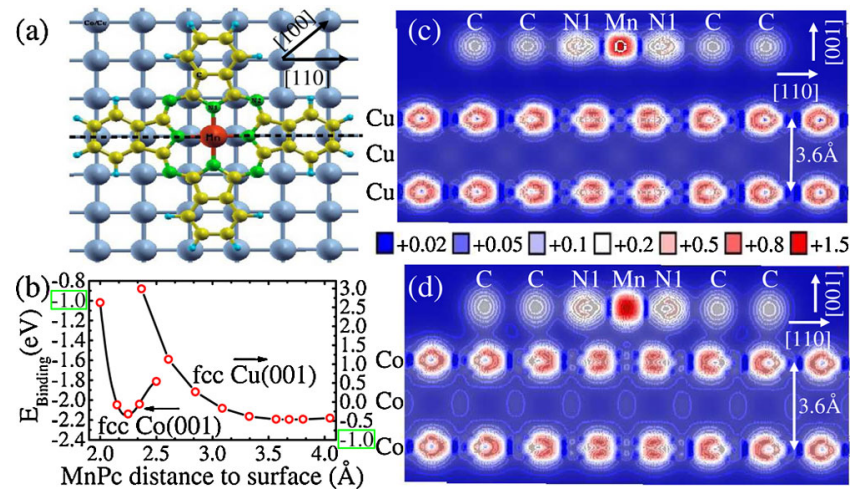

FIG. 1 (color online). (a) Adsorption geometry of MnPc on an fcc (001) surface. (b) Binding energy vs MnPc distance to the $\mathrm{Co}(001)$ and $\mathrm{Cu}(001)$ surfaces. Charge density profiles [cut in a (110) plane, along the line shown in panel (a)] for (c) $\mathrm{MnPc} / \mathrm{Cu}$ and (d) $\mathrm{MnPc} / \mathrm{Co}$ in the plane perpendicular to the substrate. 
the layers was $1.4 \mathrm{~nm}$. Each supercell consisted of 249 atoms, including the 57 atoms of MnPc. Since Co was epitaxially grown on $\mathrm{Cu}$, we used the fcc lattice parameter of $3.6 \AA$ for both $\mathrm{Co}$ and $\mathrm{Cu}$. The adsorption geometry of the Pc molecule on the (001) fcc surface is shown in Fig. 1(a). Increasing the vacuum spacing did not significantly alter the electronic structure or the total energy.

Both our experimental data and theory indicate that $\mathrm{MnPc}$ molecules lie flat onto the substrate, in agreement with a report on a similar system [9]. According to our $a b$ initio calculations, the molecule adsorbs in the bridge position for both $\mathrm{Cu}(001)$ and $\mathrm{Co}(001)$. We found that other positions, like top and hollow, are less favorable. Figure 1(b) shows how the binding energy of the system is minimized when the MnPc molecule lies $\sim 2.25 \AA$ away from the Co surface and $\sim 3.60 \AA$ away from the $\mathrm{Cu}$ surface. The distance between the $\mathrm{Mn}$ and the first $\mathrm{Co}$ neighbors is $2.58 \AA$, close to the $2.51 \AA$ separating two Co first neighbors. We find binding energies of $2.2 \mathrm{eV}$ for $\mathrm{MnPc} / \mathrm{Co}$ and $0.5 \mathrm{eV}$ for $\mathrm{MnPc} / \mathrm{Cu}$. This shows that $\mathrm{MnPc}$ is more strongly bonded to $\mathrm{Co}$ than to $\mathrm{Cu}$, which can be visualized in Fig. 1(c) and 1(d) through the charge density contours across the two interfaces along the direction indicated in Fig. 1(a). Indeed, while MnPc does not share much charge density with the surface $\mathrm{Cu}$ atoms, contours that propagate from $\mathrm{Co}$ atoms to the $\mathrm{C}, \mathrm{N}$ and $\mathrm{Mn}$ molecular sites indicate the formation of covalent bonding and antibonding states. Thus, despite the same adsorption geometry, the molecular distance to the surface and the nature of the ensuing bond strongly depend on the couple molecule or surface that is considered [15]: MnPc is physisorbed onto $\mathrm{Cu}(001)$, while it is chemisorbed onto $\mathrm{Co}(001)$. Including van der Waals (vdW) interactions could shrink the MnPc-Cu distance to $\sim 3.2 \AA$ [16], but this still remains too large for chemisorption, in agreement with our experimental observation of a mostly unperturbed molecule (see hereafter). The already low MnPc-Co distance should be mostly unaffected by vdW interactions.

We present in Fig. 2 XAS spectra acquired at the $\mathrm{N} K$ edge with the photon's electric field out of the molecular plane [Fig. 2(a), N $1 s \rightarrow \pi^{*}$ ] [17]; and in the plane [Fig. 2(b), N $1 s \rightarrow \sigma^{*}$ ]. For both experimental geometries,
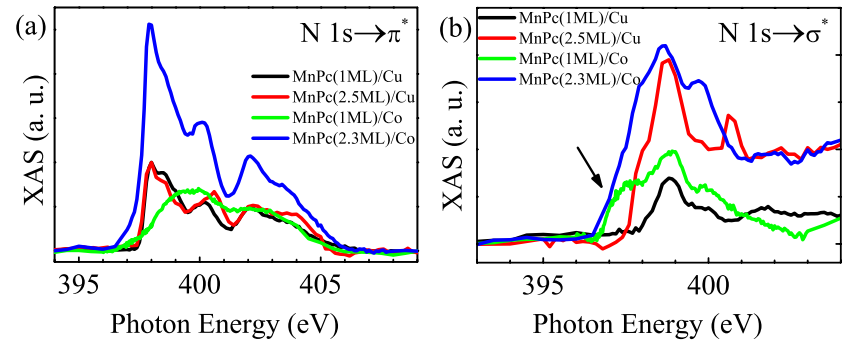

FIG. 2 (color online). XAS spectra at the $\mathrm{N} K$ edge acquired at $T=300 \mathrm{~K}$ and $H=0$ for $\operatorname{MnPc}(1 ; 2.5 \mathrm{ML}) / \mathrm{Cu}$ and $\operatorname{MnPc}(1 ; 2.3 \mathrm{ML}) / \mathrm{Co}$ so as to probe (a) $\mathrm{N} 1 s \rightarrow \pi^{*}$ and (b) $\mathrm{N}$ $1 s \rightarrow \sigma^{*}$ transitions. the spectra acquired for $1 \mathrm{ML}$ and $2.5 \mathrm{ML} \mathrm{MnPc}$ thicknesses on $\mathrm{Cu}(001)$ are essentially the same. This shows that the electronic properties on the $\mathrm{N}$ site are the same whether at, or away from, the $\mathrm{MnPc} / \mathrm{Cu}$ interface, in agreement with the aforementioned low impact on the molecule's electronic properties of physisorption onto $\mathrm{Cu}(001)$. For the $\mathrm{MnPc} / \mathrm{Co}$ system, the XAS spectra acquired for 2.3 ML MnPc on $\mathrm{Co}(001)$ share some of the essential features of the XAS spectra found on $\mathrm{Cu}(001)$. However, referring to the $\mathrm{N} 1 s \rightarrow \sigma^{*}$ transitions of Fig. 2(b), the edge is also weighed to lower energies by a vanishing contribution. When the MnPc thickness is decreased to $1 \mathrm{ML}$, this contribution now dominates the $\mathrm{N}$ pre-edge region, such that the onset of the $\mathrm{N}$ edge is effectively shifted by $\sim 1 \mathrm{eV}$ to lower energies. This is the manifestation on the XAS spectra of the formation of bonding states between $\mathrm{N}$ and $\mathrm{Co}$, and their impact on the $\sigma$-dominated intramolecular electronic structure. Similarly, spectra acquired for $\mathrm{MnPc}(1 \mathrm{ML}) / \mathrm{Co}$ that probe the $\mathrm{N} 1 s \rightarrow \pi^{*}$ transitions are strongly modified with a spectral weight to the center of the transition.

We present in Fig. 3 the $\sigma$ and $\pi$ N site-resolved partial density of states (PDOS) for the MnPc free molecule and $\mathrm{MnPc} / \mathrm{Cu}(001)$ and $\mathrm{MnPc} / \mathrm{Co}(001)$ interfaces. To identify what part of the electronic properties reflects the Mn-N bonds within the molecular plane, we have also plotted the $\sigma$ - and $\pi$-resolved N PDOS for the metal-free $\mathrm{H}_{2} \mathrm{Pc}$ molecule. The spectral tightening of the $\mathrm{N} \pi$ PDOS for $\mathrm{MnPc} / \mathrm{Co}$ compared to $\mathrm{MnPc} / \mathrm{Cu}$ is in line with experimental results [see Fig. 2(a)], underscoring the impact of the molecular chemisorption to Co through out-of-plane $\mathrm{N}$ bonds, and in contrast to physisorption onto $\mathrm{Cu}$. However, the complexity of the spectra complicates any further analysis. We now turn in Fig. 3(b), to $\sigma$-resolved PDOS, to explain the presence of the XAS edge onset shift for $\mathrm{MnPc}(1 \mathrm{ML}) / \mathrm{Co}$. Indeed, the N PDOS for the MnPc free molecule reveals the presence of molecular orbitals (MOs) starting precisely just above the Fermi level $E_{F}$. Since these MOs are absent from the $\mathrm{H}_{2} \mathrm{Pc} \mathrm{N}$ PDOS, we conclude that these MOs describe the Mn-N bond in the plane of the $\mathrm{MnPc}$ molecule. Yet, while these MOs remain essentially unchanged when the MnPc molecule is physisorbed onto $\mathrm{Cu}(001)$, an additional feature in the $\sigma$ PDOS appears at
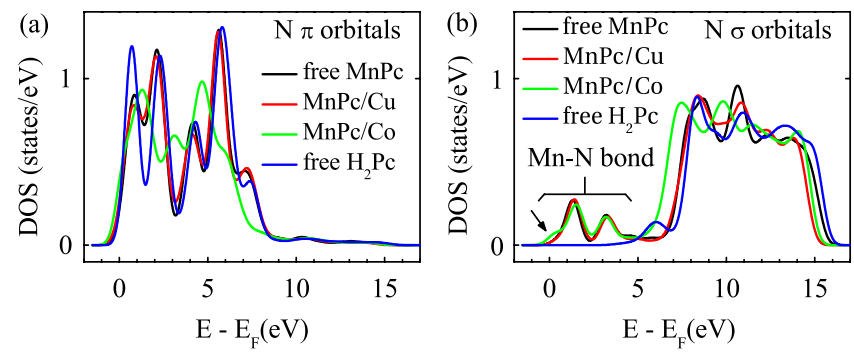

FIG. 3 (color online). Unoccupied N PDOS for (a) $\pi$ and (b) $\sigma$ orbitals of free $\mathrm{MnPc}$ and $\mathrm{H}_{2} \mathrm{Pc}$ molecules, $\mathrm{MnPc} / \mathrm{Cu}$ and $\mathrm{MnPc} / \mathrm{Co}$. 

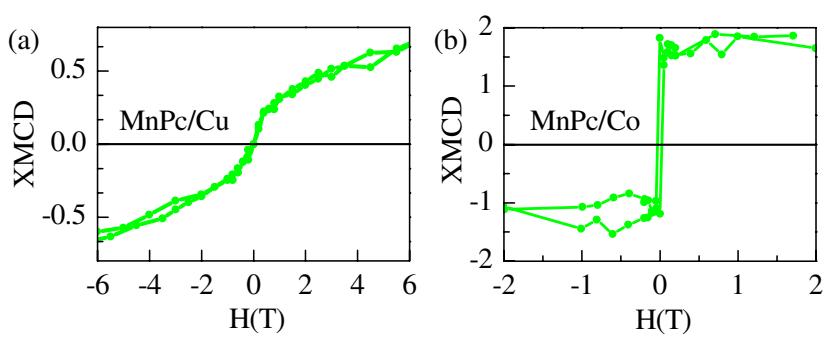

FIG. 4 (color online). XMCD $H$ dependence at the $\mathrm{Mn} L_{3}$ edge $(E=640 \mathrm{eV})$ for (a) $\operatorname{MnPc}(2.5 \mathrm{ML}) / \mathrm{Cu}$ and (b) $\operatorname{MnPc}(1 \mathrm{ML}) / \mathrm{Co}$.

lower energies when the $\mathrm{MnPc}$ molecule is deposited onto $\mathrm{Co}(001)$ [see arrow on Fig. 3(b)], in agreement with experiment (see Fig. 2). These two bonding scenari also have consequences on the electronic and magnetic properties of the central Mn molecular site (see the supplementary information [19]).

We now discuss the magnetic field dependence of the x-ray magnetic circular dichroism (XMCD) amplitude at the Mn $L_{3}$ edge. For MnPc/Co [Fig. 4(b)], we observe a square loop that coincides with that found for Co, implying a FM coupling between the Mn molecular site and the Co substrate as reported in similar cases $[8,20]$. On the other hand, for $\mathrm{MnPc} / \mathrm{Cu}$, the unsaturated loop resembles the Brillouin dependence of a paramagnetic ion [see Fig. 4(a)].

To explain these stark differences in the XAS spectra for $\mathrm{MnPc}$ on $\mathrm{Cu}(001)$ and $\mathrm{Co}(001)$ surfaces, we present in Fig. 5 the calculated spin-polarized partial densities of states (PDOS) for the two systems, and compare them to the Mn PDOS found for a free MnPc molecule. Regarding $\mathrm{MnPc}$ on $\mathrm{Cu}$, we find little hybridization near $E_{F}$ between the $\mathrm{Mn} 3 d$ and $\mathrm{Cu} 3 d$ states: the DOS of $\mathrm{MnPc}$ on $\mathrm{Cu}(001)$ is close to that of a free molecule, implying that the $\mathrm{MnPc} / \mathrm{Cu}$ interface is magnetically inactive in agreement with the XMCD field dependence. On the other hand, due to the exchange splitting, the Co $3 d$ band exhibits a substantial energy overlap with the Mn $3 d$ PDOS. Because of the resulting hybridization between $\mathrm{Mn} 3 d$ and Co $3 d$ states, the majority spin Mn PDOS is smeared to resemble that of $\mathrm{Co}$, while the multiplet features in the minority spin Mn PDOS are broadened. While the calculated magnetic
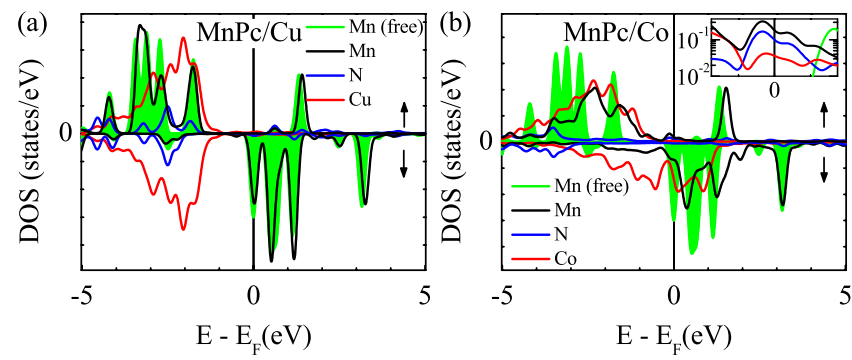

FIG. 5 (color online). The spin-polarized PDOS of Mn within the free $\mathrm{MnPc}$ molecule is compared with the $\mathrm{Mn}, \mathrm{N}$ and $\mathrm{Cu}(\mathrm{Co})$ spin-polarized PDOS for $\mathrm{MnPc}$ on (a) $\mathrm{Cu}(001)$ and (b) $\mathrm{Co}(001)$. Inset to panel (b): spin $\uparrow \mathrm{PDOS}$ of $\mathrm{MnPc} / \mathrm{Co}$ near $E_{F}$. moment of the $\mathrm{Mn}$ site for the $\mathrm{MnPc}$ molecule on $\mathrm{Cu}$ is the same as that for a free molecule $\left(3.47 \mu_{B}\right)$, it is reduced for a MnPc molecule on Co $\left(2.90 \mu_{B}\right)$. A similar reduction in magnetic moment was observed by $\mathrm{Hu}$ et al. for $\mathrm{MnPc}$ or $\mathrm{FePc}$ on $\mathrm{Au}(111)$ surface, while a slight increase of the magnetization of Fe octaethylporphyrin chloride (OEP) was obtained by Wende et al. $[8,15]$. These results imply that the $\mathrm{MnPc} / \mathrm{Co}$ interface is magnetically active, in agreement with the experimentally observed FM coupling. Our experiment and theory thus depict the MnPc molecule as physisorbed onto $\mathrm{Cu}(001)$ with the electronic properties of a free molecule, thus leading to a magnetically inactive interface. On the other hand, the chemisorption of MnPc onto $\mathrm{Co}(001)$ at a $2.25 \AA$ distance leads to out-of-plane bonds between the Mn, $\mathrm{N}$ and $\mathrm{C}$ molecular sites and $\mathrm{Co}$ surface sites as underscored in the data of Figs. 2-5. The small change in the in-plane Mn-N MO, which would take part in an indirect FM interaction between $\mathrm{Mn}$ and Co, is overshadowed by the large changes to out-of-plane MOs. As explained in [19], chemisorption could explain the alteration of the multiplet structure at the $\mathrm{Mn} L_{3}$ edge. We therefore attribute the observed FM coupling between Co and Mn to a direct coupling, as suspected by Scheybal et al. regarding similar molecules [20]. This is in contradiction to Wende et al. who reported for a similar molecule, namely OEP, a distance [8] that was too large [21] and put forward an indirect coupling mechanism mediated by the $\mathrm{N}$ surrounding the central element [8].

To assess the spintronic impact of this magnetically active $\mathrm{MnPc} / \mathrm{Co}$ interface, we now discuss spin-polarized transport channels at $E_{F}$. We present in Fig. 6 spinpolarized spatial maps, taken along the direction shown in Fig. 1(a), of the DOS, integrated over $E_{F}<E<E_{F}+$ $0.15 \mathrm{eV}$ so as to realistically reflect the effects of broadening. Generally, the molecule's spin $\downarrow$ DOS is one order of magnitude larger than the spin $\uparrow$ DOS, in line with the Co DOS. In particular, the spin $\downarrow$ DOS on the Mn site is parallel with the Co spin $\downarrow$ DOS, in agreement with the observation of FM coupling discussed above. We find that a small spin $\uparrow$ DOS bridges the $\mathrm{Mn}, \mathrm{N}$ and $\mathrm{C}$ sites to the Co sites, in

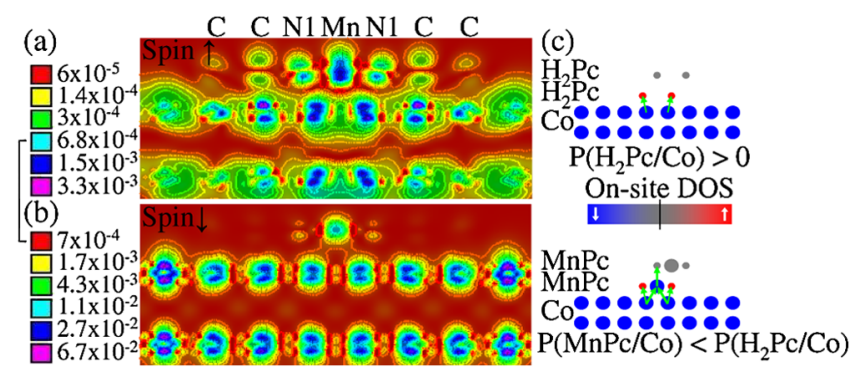

FIG. 6 (color online). Charge density profiles over the energy range $E_{F}<E<E_{F}+0.15 \mathrm{eV}$ of $\mathrm{MnPc} / \mathrm{Co}(001)$ for spin (a) $\uparrow$ and (b) $\downarrow$ charge carriers. (c) On-site DOS at the $\mathrm{H}_{2} \mathrm{Pc} / \mathrm{Co}$ and $\mathrm{MnPc} / \mathrm{Co}$ interfaces. Green arrows indicate wave function hybridization toward decay into the hypothetical Pc film. Only the central and N1 molecular sites are represented for clarity. 
agreement with data from the inset to Fig. 5(b). The spin $\downarrow$ channel is, however, driven by a strong metallic bridge between Mn and Co. Referring to Fig. 6(c), if the Mn-Co bridge is absent, i.e., in the case of a metal-free $\mathrm{H}_{2} \mathrm{Pc} / \mathrm{Co}$ interface, then weak metallic conduction in the spin $\uparrow$ channel will occur from the Co sites to the $\mathrm{C}$ and $\mathrm{N}$ sites at the interface. In analogy to wave function hybridization arguments made for inorganic spintronics [3], this should lead to a positive interfacial spin polarizarion $P_{\text {int }}>0$. Relative to this case, if the bridge is present, e.g., for the $\mathrm{MnPc} / \mathrm{Co}$ interface, the many weak spin $\uparrow$ channels mediated by the $\mathrm{N}$ and $\mathrm{C}$ sites [Fig. 6(a)] will compete with a strong Co-Mn spin $\downarrow$ conduction channel [Fig. 6(b)] [22] to define the amplitude and sign of $P_{\text {int }}$, whether through metallic or localized interface states [24]. See [19] for a more rigorous demonstration of this spintronics control over a molecular magnetic junction (e.g., in a scanning tunneling microscopy experiment; STM).

We now qualitatively discuss the impact of our results on real devices with a finite semiconductor thickness. As discussed previously (see Fig. 2), the properties of additional layers away from the interface should resemble those of bulk MnPc. As we argue in [19], both the highest occupied molecular orbital and lowest unoccupied molecular orbital of bulk MnPc should be weighed with $\mathrm{Mn}$ character. This means that wave functions with Mn character shall efficiently transmit (see e.g., [1,2]) across bulk MnPc. This implies that the control over $P_{\text {int }}$ through the presence of $\mathrm{Mn}$ in $\mathrm{MnPc}$ relative to $\mathrm{H}_{2} \mathrm{Pc}$ shall carry over to an effective spin polarization for elastic tunneling (See [19] for more information). These results thus explicitly demonstrate how an interface state can drive the spin-polarized tunneling properties of a device, as was recently argued to account for large values of magnetoresistance across an organic magnetic tunnel junction [10]. In addition, our results also show how to control the spintronic response of this interface state. To extend this argument to the diffusive transport regime, one should also take into account the organic semiconductor resistivity and spin diffusion length [25], which depends on the nature of transport [10].

In conclusion, we have experimentally and theoretically examined the consequences for molecular spintronics of the adsorption mechanisms that bind $\mathrm{MnPc}$ to model surfaces. While $\mathrm{MnPc}$ is physisorbed onto $\mathrm{Cu}(001)$ and retains the electronic structure of a free molecule, chemisorption onto $\mathrm{Co}(001)$ leads to covalent bonding and direct ferromagnetic coupling between the molecule's Mn site and the Co surface sites. The ensuing metallic bridge across the two sites effectively shifts the onset of tunneling onto the molecule, which is thus part of the electrode. We find that removing the Mn site from the Pc molecule will change the interfacial tunneling spin polarization to be observed in a STM experiment, and also in principle the effective spin polarization of a tunnel junction interface. This research underscores the tremendous potential to craft the interfacial electronic properties for molecu- lar spintronic devices with novel responses, and, in particular, to achieve control over the sign of the interfacial tunneling spin polarization through only a subtle modification of the interface structure. This ease of spintronics control is unparalleled in inorganic spintronics [26].

We are grateful to C. Barraud, P. Seneor, and R. Mattana for sharing preliminary magnetotransport data. We thank F. Gautier for stimulating discussions, F. Nolting for experimental support and B. Muller for technical support. S. Javaid is partly funded by the Pakistani government (HEC). We acknowledge financial support from the the EC Sixth Framework Program (NMP3-CT-2006-033370), the CNRS-PICS Program No. 5275 and the French ANR (ANR-06-NANO-033-01; $\quad$ ANR-06-NANO-053-01). Calculations were performed using HPC resources from GENSI-CINES (gemm1001).

[1] W. H. Butler et al., Phys. Rev. B 63, 054416 (2001).

[2] M. Bowen et al., Phys. Rev. B 73, 140408(R) (2006).

[3] E. Y. Tsymbal, O. M. Mryasov, and P. R. LeClair, J. Phys. Condens. Matter 15, R109 (2003).

[4] M. Bowen et al., Phys. Rev. B 73, 012405 (2006).

[5] T. S. Santos et al., Phys. Rev. Lett. 98, 016601 (2007).

[6] J. R. Petta, S. K. Slater, and D. C. Ralph, Phys. Rev. Lett. 93, 136601 (2004).

[7] Z. H. Xiong et al., Nature (London) 427, 821 (2004).

[8] H. Wende et al., Nature Mater. 6, 516 (2007).

[9] A. F. Takacs et al., Phys. Rev. B 78, 233404 (2008).

[10] C. Barraud et al. (private communication); C. Barraud et al., Nature Phys. (in press).

[11] A. R. Rocha et al., Nature Mater. 4, 335 (2005).

[12] P. Giannozzi et al., J. Phys. Condens. Matter 21, 395502 (2009).

[13] J. P. Perdew, K. Burke, and M. Ernzerhof, Phys. Rev. Lett. 77, 3865 (1996).

[14] D. Vanderbilt, Phys. Rev. B 41, 7892 (1990).

[15] Zhenpeng Hu et al., J. Phys. Chem. C 112, 13650 (2008).

[16] N. Atodiresei et al., Phys. Rev. Lett. 102, 136809 (2009).

[17] We achieved out-of-plane XAS sensitivity by tilting the sample by $50^{\circ}$ w.r.t. the photon beam. Since only N $1 \mathrm{~s} \rightarrow$ $\sigma^{*}$ transitions occur in the 407-417 eV energy range [18], we have subtracted the spectra for $\mathrm{N} 1 s \rightarrow \sigma^{*}$ transitions, acquired at $0^{\circ}$, from those acquired at $50^{\circ}$.

[18] I. Biswas et al., J. Chem. Phys. 126, 174704 (2007).

[19] See supplementary material at http://link.aps.org/ supplemental/10.1103/PhysRevLett.105.077201.

[20] A. Scheybal et al., Chem. Phys. Lett. 411, 214 (2005).

[21] Md. Ehesan Ali, B. Sanyal, and P. M. Oppeneer, J. Phys. Chem. C 113, 14381 (2009).

[22] Depending on the Co-Mn bridge and overall interface resistances, wave function hybridization will reflect either the Co-Mn bond or the Mn bond to the spin-unpolarized N1 site on the second molecular layer [23].

[23] Xi Chen et al., Phys. Rev. Lett. 101, 197208 (2008).

[24] E. Y. Tsymbal et al., Phys. Rev. Lett. 90, 186602 (2003).

[25] A. Fert and H. Jaffrès, Phys. Rev. B 64, 184420 (2001).

[26] J. M. De Teresa et al., Science 286, 507 (1999). 